\title{
Tolerance to drug-induced cell death favours the acquisition of multidrug resistance in Leishmania
}

\author{
W Moreira ${ }^{1}$, P Leprohon ${ }^{1}$ and M Ouellette ${ }^{*, 1}$
}

The control of the protozoan parasite Leishmania relies on few drugs with unknown cellular targets and unclear mode of action. Several antileishmanials, however, were shown to induce apoptosis in Leishmania and this death mechanism was further studied in drug-sensitive and drug-resistant Leishmania infantum. In sensitive parasites, antimonials (SbIII), miltefosine (MF) and amphotericin B (AMB), but not paromomycin (PARO), triggered apoptotic cell death associated with reactive oxygen species (ROS). In contrast, Leishmania mutants resistant to SbIII, MF or AMB not only failed to undergo apoptosis following exposure to their respective drugs, but also were more tolerant towards apoptosis induced by other antileishmanials, provided that these killed Leishmania via ROS production. Such tolerance favored the rapid acquisition of multidrug resistance. PARO killed Leishmania in a non-apoptotic manner and failed to produce ROS. PARO resistance neither protected against drug-induced apoptosis nor provided an increased rate of acquisition of resistance to other antileishmanials. However, the PARO-resistant mutant, but not SbIII-, MF- or AMB-resistant mutants, became rapidly cross-resistant to methotrexate, a model drug also not producing ROS. Our results therefore link the mode of killing of drugs to tolerance to cell death and to a facilitated emergence of multidrug resistance. These findings may have fundamental implications in the field of chemotherapeutic interventions.

Cell Death and Disease (2011) 2, e201; doi:10.1038/cddis.2011.83; published online 1 September 2011

Subject Category: Cancer

The bactericidal mode of action of antibiotics has recently been shown to depend less on their primary target, and more on the induction of common downstream effector mechanisms leading to cell death. Indeed, the generation of oxidants has been a unifying step of such common death mechanisms (reviewed in Kohanski et al. ${ }^{1}$ ). Similarly, the induction of apoptosis by several anticancer drugs has been suggested to occur through the generation of oxidants, and a characteristic feature of drug-resistant neoplasic cells seems to be their ability to evade drug-induced apoptosis. ${ }^{2,3}$ These studies suggested a causal link between evolutionary-conserved cellular events leading to death and the mode of action of cytotoxic compounds, and highlighted the importance of understanding the mode of action of drugs in order to understand and prevent the emergence of drug resistance.

Apoptotic features have also been reported in the eukaryotic parasite Leishmania following exposure to several drugs. ${ }^{4-8}$ Leishmania are unicellular parasites responsible for a wide range of human diseases ranging from self-healing cutaneous lesions to fatal visceral infections. Anti-Leishmania therapy relies on the use of a handful number of drugs including pentavalent antimony (SbV) - a prodrug that needs to be reduced to its trivalent state (SbIII) in order to acquire antiparasitic activity, the polyene antibiotic amphotericin B (AMB) and the phosphocholine analog miltefosine (MF). ${ }^{9} \mathrm{~A}$ phase 3 clinical trial has also been successfully completed for the aminoglycoside paromomycin (PARO), ${ }^{10}$ leading to its approval for the treatment of visceral leishmaniasis in India. Although the mechanisms responsible for the anti-Leishmania activities of these compounds have not yet been clearly established, their primary targets are thought to differ substantially. Indeed, evidence suggests that antimony have a rather complex mode of action, acting at the level of multiple cellular targets. ${ }^{11-18}$ The leishmanicidal activity of MF has been associated with perturbation of the metabolism of alkylphospholipids, and the biosynthesis of alkyl-anchored glycolipids and glycoproteins. ${ }^{19,20}$ The cytotoxic activity of AMB has been associated with its high affinity for ergosterol, ${ }^{21}$ the main sterol in the plasma membrane of fungi and Leishmania. ${ }^{22}$ Finally, PARO, which interferes with protein synthesis by binding to ribosomes in bacteria, was also shown to affect translation in Leishmania, ${ }^{23}$ in addition to alter the composition and fluidity of the plasma membrane of the parasite. ${ }^{24,25}$

Most antileishmanials were shown to induce apoptosis features in Leishmania, which include an increase in intracellular calcium levels, an accumulation of reactive oxygen species (ROS), a drop of the mitochondrial membrane potential $(\Delta \psi \mathrm{m})$, genomic DNA degradation, exposure of phosphatidyl serine and induction of caspase-like activity. $7,16,17,26,27$ Interestingly, the antimony-resistant phenotype of field isolates was reported to be accompanied by a decreased propensity to undergo apoptosis following exposure to either SbIII, MF or AMB. ${ }^{7}$ Therefore, it is possible that the common mechanisms involved in cell killing described for

\footnotetext{
${ }^{1}$ Centre de Recherche en Infectiologie du CHUL, Université Laval, Québec, Quebec, Canada

${ }^{*}$ Corresponding author: M Ouellette, Centre de Recherche en Infectiologie du CHUL, Université Laval, 2705 Laurier Boulevard, RC709, Québec, Quebec G1V 4G2, Canada. Tel: + 1418654 2705; Fax: + 1418654 2715; E-mail: Marc.Ouellette@crchul.ulaval.ca

Keywords: Leishmania; apoptosis; multidrug resistance; reactive oxygen species

Abbreviations: $\Delta \psi \mathrm{m}$, mitochondrial membrane potential; AMB, amphotericin B; DCFDA, dichlorofluorescein diacetate; GSH, glutathione; MF, miltefosine; MitoSOX, red mitochondrial superoxide indicator; MTX, methotrexate; PARO, paromomycin; ROS, reactive oxygen species; TMRE, tetramethylrhodamine ethyl ester Received 21.3.11; revised 11.7.11; accepted 25.7.11; Edited by A Stephanou
} 
bactericidal antibiotics might hold true for leishmanicidal compounds and that a decrease in sensitivity to one drug could favor the development of resistance against other drugs.

In this study, we sought to determine the putative apoptotic features induced by SbIII, MF, AMB and PARO in Leishmania infantum drug-sensitive and drug-resistant parasites, and to assess whether an increased tolerance to apoptosis might accompany the selection of drug resistance. Our results are suggesting two types of drug-induced death in Leishmania, and are linking the mode of killing to tolerance to cell death and to a facilitated emergence of multidrug resistance.

\section{Results}

Drug-induced accumulation of ROS in the Leishmania mitochondria. As several drugs were shown to induce apoptosis in Leishmania, we first studied the accumulation of ROS upon drug exposure in the context of drug sensitivity and resistance. The $L$. infantum Sb2000.1, MF200.5, AMB1000.1 and PAR01200.1 cell lines were selected for resistance to SbIII, MF, AMB and PARO, respectively, in a stepwise manner until they reached at least a 10-fold increased resistance compared with the parental clonal strain (Table 1). None of the mutants showed marked cross-resistance, with the exception of the Sblll- and PARO-resistant mutants, which were five to six times hypersensitive to MF (Table 1). The accumulation of ROS was measured using the dichlorofluorescein diacetate (DCFDA) dye whose fluorescence intensity is indicative of the levels of intracellular ROS. ${ }^{28} \mathrm{~L}$. infantum-sensitive parasites subjected to increasing concentrations of Sblll, MF and AMB exhibited a dose-dependent increase in DCFDA fluorescence signals (Figure 1), confirming increased ROS production upon exposure to these antileishmanials. Pentamidine, another anti-Leishmania drug, also induced the accumulation of ROS in sensitive L. infantum (Supplementary Figure S1A) and so was the classical apoptosis-inducing drug staurosporine ${ }^{29}$ (Supplementary Figure S1C). In contrast, no ROS production could be measured by DCFDA fluorescence in the resistant mutants Sb2000.1, MF200.5 and AMB1000.1 exposed to the drug defining their resistance phenotype (Figure 1). PARO behave differently as it failed to induce ROS accumulation irrespective of the PARO susceptibility status of the parasites (Figure 1). This was not unique to
PARO as we found that methotrexate (MTX), a model drug often studied in Leishmania, ${ }^{30,31}$ also failed to induce ROS in Leishmania (Supplementary Figure S1B).

As the major source of oxidants in eukaryotes is the mitochondria, we used the MitoSox dye (red mitochondrial superoxide indicator) to measure the mitochondrial accumulation of superoxide, which is an indicative measure of ROS level in the mitochondria. ${ }^{32}$ As expected, SbIII, MF and AMB induced an increase in MitoSox fluorescence levels in sensitive but not resistant parasites (Figure 2a). Colocalization experiments between DCFDA and mitotracker fluorescence signals further confirmed that the mitochondria were the site of drug-induced ROS accumulation (Figure 2b). The accumulation of superoxide within the mitochondria usually results in a misregulation of cellular iron homeostasis through the oxidation of iron-sulfur clusters, which translates into the production of highly active hydroxyl radicals via the Fenton reaction. ${ }^{4,33}$ The role of iron in the drug-induced accumulation of oxidants was confirmed by the reduced level of ROS produced within the mitochondria of sensitive $L$. infantum pretreated with the iron-chelating agent deferoxamine before being exposed to increasing concentrations of SbIII, MF and AMB (Figure 2c). The same protective effect against the accumulation of ROS was observed in $L$. infantum parasites pretreated with the antioxidant glutathione (GSH) (Figure 2c).

Every experiments presented so far were performed with the more experimentally tractable promastigote insect stage of the parasite. Accordingly, we tested whether a similar druginduced ROS accumulation could be observed with the more clinically relevant intracellular amastigotes. MitoSOX-preloaded promastigotes were used to infect macrophages and the resulting intracellular sensitive parasites, but not their resistant counterparts, accumulated ROS when exposed to $\mathrm{SbV}$ (the prodrug that is converted to SbIII), MF or AMB (Figure 2d).

Tolerance to drug-induced apoptosis in drug-resistant Leishmania. As the accumulation of oxidants within the mitochondria is one of the hallmarks of apoptosis including in parasites, ${ }^{34,35}$ we tested whether other apoptotic features could be observed following exposure to different antileishmanials. We monitored changes in the $\Delta \psi \mathrm{m}$ using the tetramethylrhodamine ethyl ester (TMRE) dye, ${ }^{7}$ a cationic fluorescent molecule whose accumulation within the mitochondria is an indicative measure of the $\Delta \psi \mathrm{m}$, and tested for genomic DNA degradation by gel electrophoresis.

Table 1 Susceptibility of $L$. infantum WT and drug-resistant mutants to SbIII, MF, AMB, PARO and MTX

\begin{tabular}{|c|c|c|c|c|c|}
\hline & Sblll ( $\mu M)$ & MF $(\mu \mathrm{M})$ & AMB $(\mu \mathrm{M})$ & PARO $(\mu \mathrm{M})$ & MTX $(\mu \mathrm{M})$ \\
\hline $\begin{array}{l}\text { WT } \\
\text { Sb2000.1 } \\
\text { MF200.5 } \\
\text { AMB1000.1 } \\
\text { PARO1200.1 } \\
\text { MTX1000.3 }\end{array}$ & $\begin{array}{c}49.6 \pm 9.6 \\
>2000^{\star *} \\
116 \pm 24^{\star} \\
97 \pm 3^{\star} \\
50 \pm 1.4 \\
\text { ND }\end{array}$ & $\begin{array}{c}16.3 \pm 5.4 \\
3.3 \pm 0.5^{\star} \\
>200^{\star *} \\
21 \pm 2.6 \\
2.8 \pm 0.4^{\star} \\
N D\end{array}$ & $\begin{array}{c}0.040 \pm 0.013 \\
0.045 \pm 0.028 \\
0.055 \pm 0.017 \\
>1000^{\star *} \\
0.040 \pm 0.012 \\
\mathrm{ND}\end{array}$ & $\begin{array}{c}80.9 \pm 39.3 \\
142.8 \pm 15.7^{\star} \\
104.3 \pm 21.4 \\
110.7 \pm 24.4 \\
>1680^{\star *} \\
60 \pm 17.6\end{array}$ & $\begin{array}{c}255 \pm 23 \\
N D \\
N D \\
N D \\
212 \pm 18 \\
>1000^{\star *}\end{array}$ \\
\hline
\end{tabular}

Abbreviations: AMB, amphotericin B; MTX, methotrexate; MF, miltefosine; ND, not determined; PARO, paromomycin; WT, wild-type

${ }^{\star} P<0.05 ;{ }^{* \star} P<0.01$

$\mathrm{EC}_{50}$ were determined after $72 \mathrm{~h}$ of culture in the presence of various drug concentrations as described ${ }^{28}$

Results are the average of at least three independent experiments

Paired Student's $t$-test $\left({ }^{\star} P<0.05 ;{ }^{\star \star} P<0.01\right)$ 

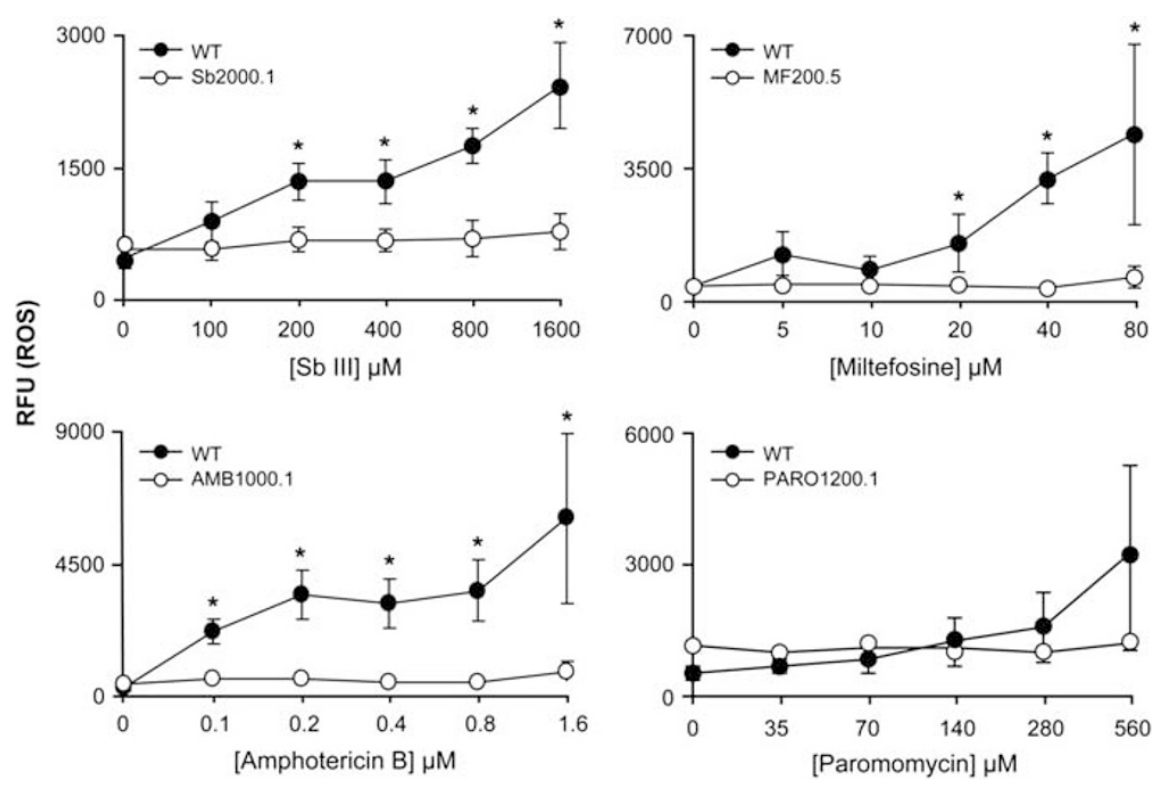

Figure 1 Drug-induced ROS in Leishmania infantum wild-type and drug-resistant mutants. Drug-induced ROS accumulation in L. infantum cells. L. infantum WT or L. infantum Sb.2000.1, MF200.5, AMB1000.1 and PAR01200.1 log-phase cultures, selected for resistance to SbIII, MF, AMB and PARO, respectively, were exposed to increasing concentrations of drugs for $48 \mathrm{~h}$. Aliquots $(500 \mu \mathrm{l})$ were washed and incubated in the presence of DCFDA for 30 min. Fluorescence was measured at $530 \mathrm{~nm}$ and normalized according to protein concentrations. Results are the average of three independent experiments. ${ }^{*} P<0.05$

A significant drop in the $\Delta \psi \mathrm{m}$ was observed in sensitive cells exposed to SbllI, MF and AMB, but not with PARO (Figure 3a). Similarly, drug-induced DNA laddering occurred in L. infantum wild-type cells treated with Sblll, MF and AMB, but not with PARO (Figure 3b). Interestingly, neither a decrease in $\Delta \psi \mathrm{m}$ nor a degradation of genomic DNA could be observed in the drug-resistant mutants exposed to their selective drugs (Figures $3 a$ and $b$ ). In contrast, the protonophore carbonyl cyanide p-trifluoromethoxy-phenylhydrazone remained potent at inducing a significant loss in $\Delta \psi \mathrm{m}$ in these mutants (Figure $3 a$ ), suggesting that the proton gradient was still intact in the resistant parasites.

Having found that SbIII, MF, AMB, but not PARO, induced several apoptosis features in drug-sensitive parasites but not in the Sb2000.1-, MF200.5- and AMB1000.1-resistant lines, respectively, we sought to understand whether a non-specific tolerance to drug-induced apoptosis occurred in these mutants. Interestingly, the Sb2000.1-, MF200.5- or AMB1000.1-resistant mutants failed to produce ROS when challenged with the drug used for their selection, and there was also a significant lack of ROS production in these mutants upon challenge with any of the ROS-inducing drugs (Figure 4a). Again, the PAR01200.1 mutant behave differently as ROS levels comparable to wild-type cells were observed in this mutant after a challenge with SbIll and even higher levels were observed after a challenge with MF or AMB (Figure $4 \mathrm{a})$. The drop in $\Delta / \mathrm{m}$ following exposure either to SbIII, MF or AMB was also lower in the Sb2000.1, MF200.5 and $A M B 1000.1$ mutants than in the sensitive cells or the PAR01200.1 mutant (Figure 4b). The modulation in ROS production and change in $\Delta \psi \mathrm{m}$ were further correlated with drug-induced laddering of genomic DNA. Indeed, no DNA laddering was induced in the Sb2000.1, MF200.5 and
AMB1000.1 mutants following exposure either to SbIII, MF or AMB, whereas the same drugs induced oligonucleosomal fragmentation in the PARO1200.1 mutant (Figure 4c). We also tested whether this phenomenon of cross-tolerance to drug-induced apoptosis occurred with intracellular amastigotes. We measured ROS in parasites infecting macrophages and we found that the Sb2000.1, MF200.5 and AMB1000.1, but not PARO1200.1, failed to produce more ROS than the control, when challenged with ROS-inducing drugs (Figure 4d). As GSH was shown to protect cells against drug-induced ROS (Figure 2c) and that GSH has been linked to Sblll resistance, ${ }^{36-39}$ we tested whether cells resistant to ROS-inducing drugs had increased levels of reduced thiols. However, analysis by HPLC failed to show a significant increase of reduced thiols in the drug-resistant mutants (results not shown).

Tolerance to drug-induced cell death favors the acquisition of multidrug resistance. We showed that the tolerance to drug-induced apoptosis in the Sb2000.1, MF200.5 and AMB1000.1 lines occurred not only against the drug initially used for the selection of resistance but also extended to any apoptosis-inducing drugs. We hypothesized that this phenomenon may facilitate the emergence of multiresistance against other drugs, provided that these shared similar killing effector mechanisms. We thus compared the rates of selection of resistant parasites by plating $5 \times 10^{6}$ drug-sensitive or drug-resistant parasites in the presence of MF, AMB or PARO at a concentration inhibiting the growth of L. infantum wild-type parasites. Sblll was omitted as the susceptibility to this drug varied between mutants (Table 1). The AMB1000.1, MF200.5, PARO1200.1 and MTX1000.3 mutants yielded from $6.9 \times 10^{4}$ to $1.3 \times 10^{5}$ colonies, and a plating efficiency of $\sim 10 \%$ when plated with their respective 
a

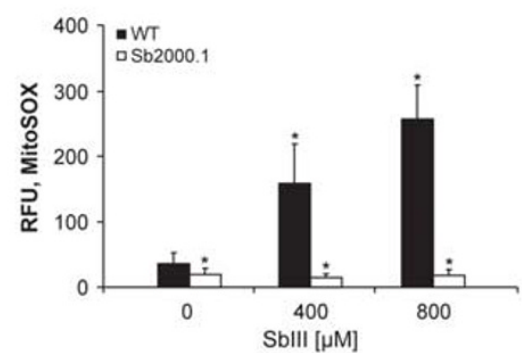

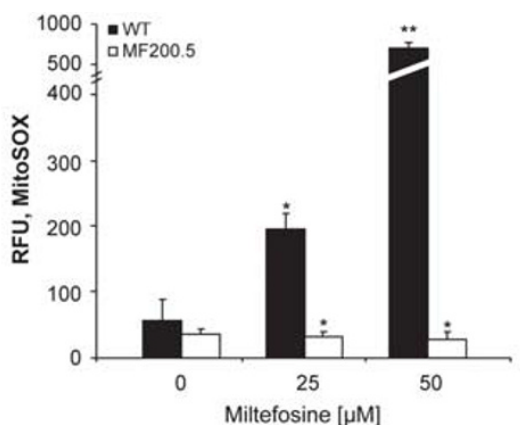

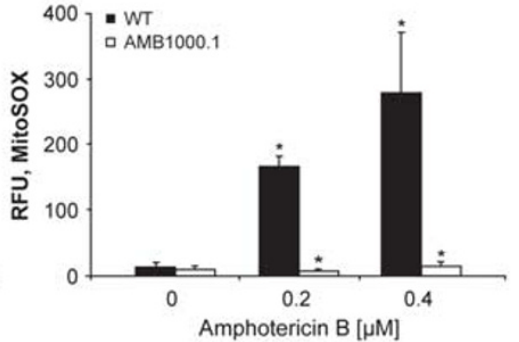

b $\quad \mathrm{WT}+$ Hepes- $\mathrm{NaCl}$
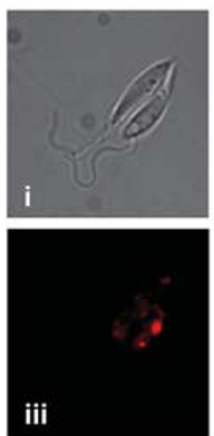
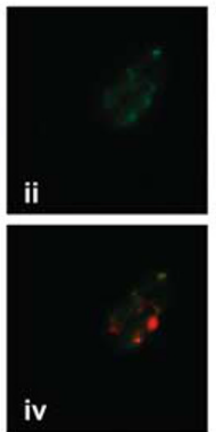

WT + SbIII $400 \mu \mathrm{M}$
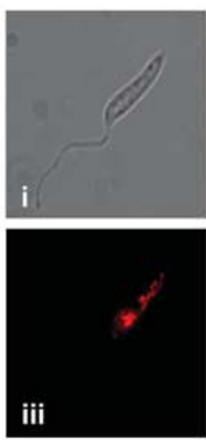
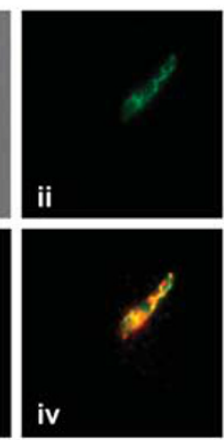

WT + Miltefosine $25 \mu \mathrm{M}$
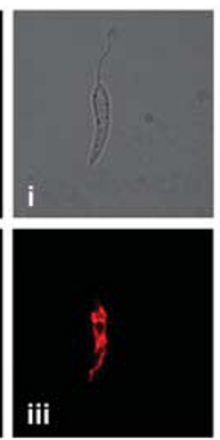
$\mathrm{WT}+$ Amphotericin B $0.2 \mu \mathrm{M}$

C

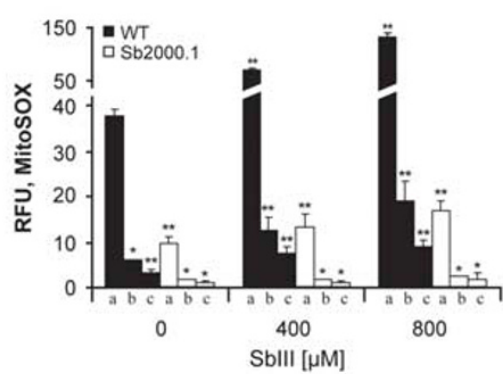

d

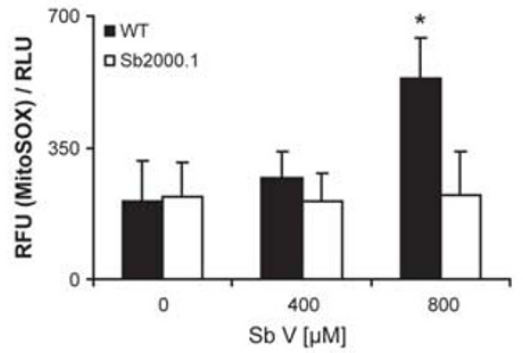

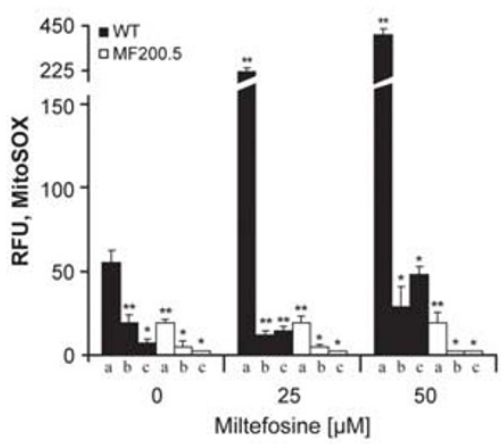

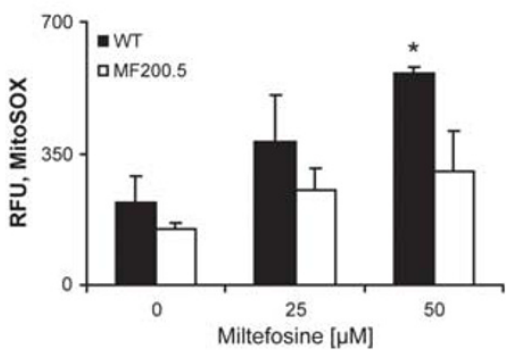

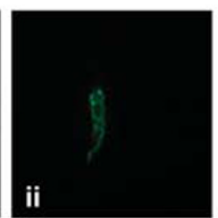

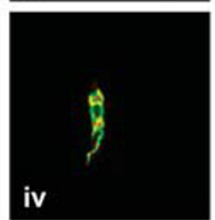

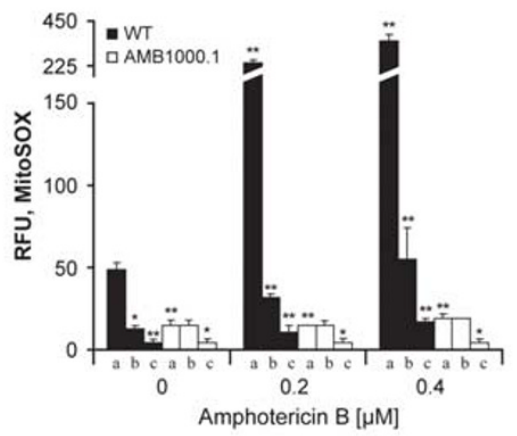
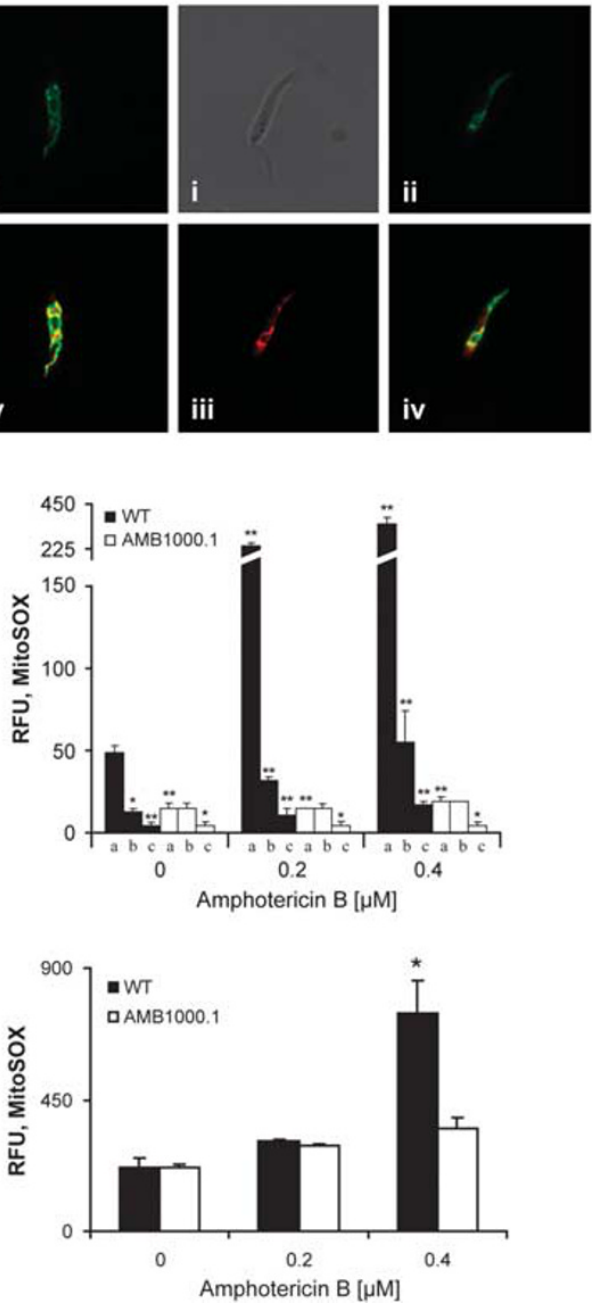

Figure 2 Drug-induced ROS accumulation in Leishmania infantum mitochondria. (a) Log-phase cultures of $L$. infantum WT or L. infantum Sb.2000.1, MF200.5 and AMB1000.1 drug-resistant mutants were preloaded with MitoSOX for $2 \mathrm{~h}$, washed and exposed to two concentrations of Sblll, MF or AMB for $48 \mathrm{~h}$. Aliquots (500 $\mu$ l) were taken, washed and the fluorescence was measured at $580 \mathrm{~nm}$. (b) ROS accumulation in Leishmania cells was visualized with the fluorescent dye DCFDA and the mitochondria-specific dye Mitotracker. Log-phase cultures were exposed to drugs for $48 \mathrm{~h}$, washed and incubated with DCFDA and Mitotracker for 30 min each. Fluorescence microscopy showed colocalization of the oxidant-dependent DCFDA and the mitochondria-specific Mitotracker in drug-treated cells. (i) Brightfield, (ii) DCFDA fluorescence, (iii) Mitotracker fluorescence, (iv) Merge. (c) Protection against drug-induced mitochondrial ROS accumulation. Log-phase cultures of WT and drug-resistant mutants were preloaded with MitoSOX for $2 \mathrm{~h}$, washed, preincubated with Hepes-NaCl (bars a), $500 \mu \mathrm{M}$ of deferoxamine (bars b), or with $2 \mathrm{mM} \mathrm{GSH}$ (bars c) for $24 \mathrm{~h}$, washed and exposed to two concentrations of SbIII, MF or AMB for $48 \mathrm{~h}$. Aliquots ( $500 \mu \mathrm{l}$ ) were taken, washed and the fluorescence was measured at $580 \mathrm{~nm}$. (d) Drug-induced ROS accumulation within intramacrophagic L. infantum. L. infantum WT (black bars), Sb2000.1 (white bars, left panel), MF200.5 (white bars, central panel) and AMB1000.1 (white bars, right panel) promastigotes containing a luciferase expression vector were preloaded with MitoSOX, and subsequently used to infect J774 macrophages. The drug-induced ROS accumulation in intracellular parasites was measured $48 \mathrm{~h}$ post infection, and fluorescence (RFU) was normalized to luciferase activity level (RLU). Results are the average of at least three independent experiments. ${ }^{*} P<0.05$; ${ }^{* *} P<0.01$ 


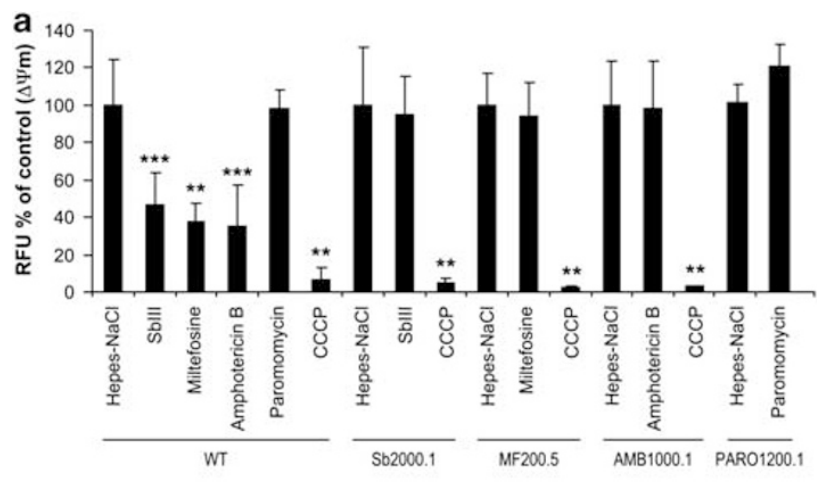

b

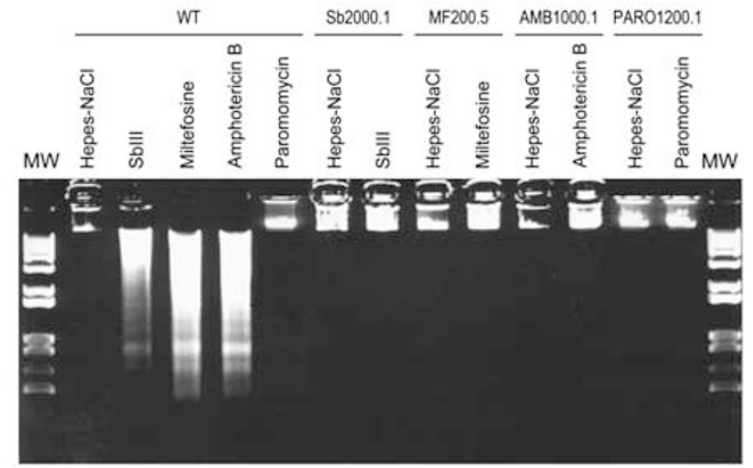

Figure 3 Drug-induced apoptosis in Leishmania. Drug-induced loss of $\Delta \psi \mathrm{m}$ (a) and gDNA laddering (b) in sensitive and resistant Leishmania cells. Log-phase cultures were exposed to drugs (Sblll, $400 \mu \mathrm{M}$; MF, $25 \mu \mathrm{M}$; AMB, $0.2 \mu \mathrm{M}$ and PARO, $280 \mu \mathrm{M}$ ) or to saline buffer (control) for $48 \mathrm{~h}$. (a) For measuring $\Delta \psi \mathrm{m}$, a 500- $\mu$ l aliquot was washed, incubated with TMRE for $30 \mathrm{~min}$, washed and the fluorescence was measured $(545 \mathrm{~nm})$. Fluorescence was normalized to protein concentrations. (b) Genomic DNA degradation was investigated by analyzing the isolated gDNA on $1.3 \%$ agarose gels. Results represent the average of at least three independent experiments and are expressed as percentage of the control. ${ }^{\star \star} P<0.01 ;{ }^{* \star} P<0.001$. MW: $1 \mathrm{~kb}$ Plus Ladder (Invitrogen)

drugs (Figure 5). In contrast, the wild-type cells did not yield any colony when plated in the presence of MF or PARO, and on an average 14 colonies were counted on the AMB plates (Figure 5a). Remarkably, the L. infantum Sb2000.1 and MF200.5 mutants generated significantly more colonies on the AMB plate than the $L$. infantum wild-type strain or the PAR01200.1 mutant (Figure 5a). Similarly, the AMB1000.1 mutant yielded significantly more colonies under MF pressure than wild-type parasites or the PARO 1200.1 mutant (Figure 5a). However, the Sb2000.1 mutant behaved unexpectedly, as few colonies were observed on MF-containing plates (Figure 5a). This is probably due to the fact that Sb2000.1 is fivefold hypersensitive to MF (Table 1).

In the presence of PARO, the MF200.5 and AMB1000.1 mutants did not yield significantly more colonies than wild-type parasites (Figure 5a). Again, the Sb2000.1 behaved in an unexpected manner, producing a very high number of colonies on PARO plates (Figure $5 \mathrm{a}$ ). This observation might be explained in part by the fact that the Sb2000.1 mutant initially exhibited a slight but significant cross-resistance to PARO (Table 1). Although PARO and MTX did not induce ROS in wild-type parasites (Figure 1 and Supplementary Figure S1), it is not clear whether similar killing effector mechanisms are shared by both drugs. Nonetheless, it is intriguing that the MTX-resistant mutant yielded more colonies than the MF200.5 mutant or wild-type parasites in the presence of PARO (Figure 5b), and that the PARO1200.1 mutant also yielded a higher number of colonies on MTXcontaining plates than wild-type or MF200.5 cells (Figure 5b).

\section{Discussion}

Our study has revealed that anti-Leishmania compounds kill by at least two different mechanisms, with SbIII, AMB, MF and most likely pentamidine killing by apoptosis, and PARO and the model drug MTX acting through another lethal mechanism. In order to be classified as apoptosis-inducing drugs, compounds were required to fulfill a number of criteria, including the induction of mitochondrial ROS, a drop in $\Delta \psi \mathrm{m}$ and genomic DNA degradation. Several studies had already reported on the role of redox pathways in the increased survival of Leishmania in the presence of SbllI, ${ }^{18,38,39}$ with iron potentiating the antileishmanial activity of Sblll. ${ }^{4}$ Our results indicate that this is probably valid for all drugs inducing ROS, as experiments under iron-depleted conditions demonstrated a key role for iron in drug-induced ROS production (Figure 2c).

The dichotomy in the mode of killing between antileishmanials was further reinforced by the observation that cells resistant to SbIII, MF and AMB were tolerant to apoptosis not only against the initial selective drug but also against all drugs sharing a similar mode of killing. This tolerance is likely to arise by alterations in shared death pathways. Obviously, alterations in these pathways do not lead to high-level crossresistance (Table 1) but can contribute to a decreased propensity to undergo apoptosis, thereby favouring the emergence of more specific resistance mechanisms. For example, although no specific mutations leading to a high level of AMB resistance are present in Sb2000.1 or MF200.5 cells (Table 1), an alteration in a death pathway allowing tolerance to AMB-induced apoptosis may likely be shared by these mutants (Figure 4). In contrast, as PARO kills through a different mechanism, we did not observe tolerance to Sblll-, MF- or AMB-induced apoptosis in the PARO-resistant mutant (Figure 4). We are currently searching for phenotypic features related to PARO and MTX killing as this would allow to test whether non-ROS-producing drugs constitute a distinct class of unrelated compounds that also share similar killing mechanisms.

Such tolerance to drug-induced killing accelerated the emergence of cross-resistance. Indeed, the SbIII-, MF- and AMB-resistant mutants, but not the PARO-resistant mutant, became more easily cross-resistant to the apoptosis-inducing drugs SbIII, MF and AMB. Conversely, the PARO- and MTXresistant mutants acquired cross-resistance to the non-ROSproducing drugs more easily. Two exceptions were noted, both involving the Sb2000.1 mutant when plated with either MF or PARO. This could possibly be explained by collateral hypersusceptibility or cross-resistance already present in this mutant (Table 1), where a mutation leading to resistance or susceptibility may supersede alteration(s) in a death pathway when cross-resistance is selected. As Sblll interferes with the thiol redox metabolism, ${ }^{18}$ it is possible that oxidant-induced 

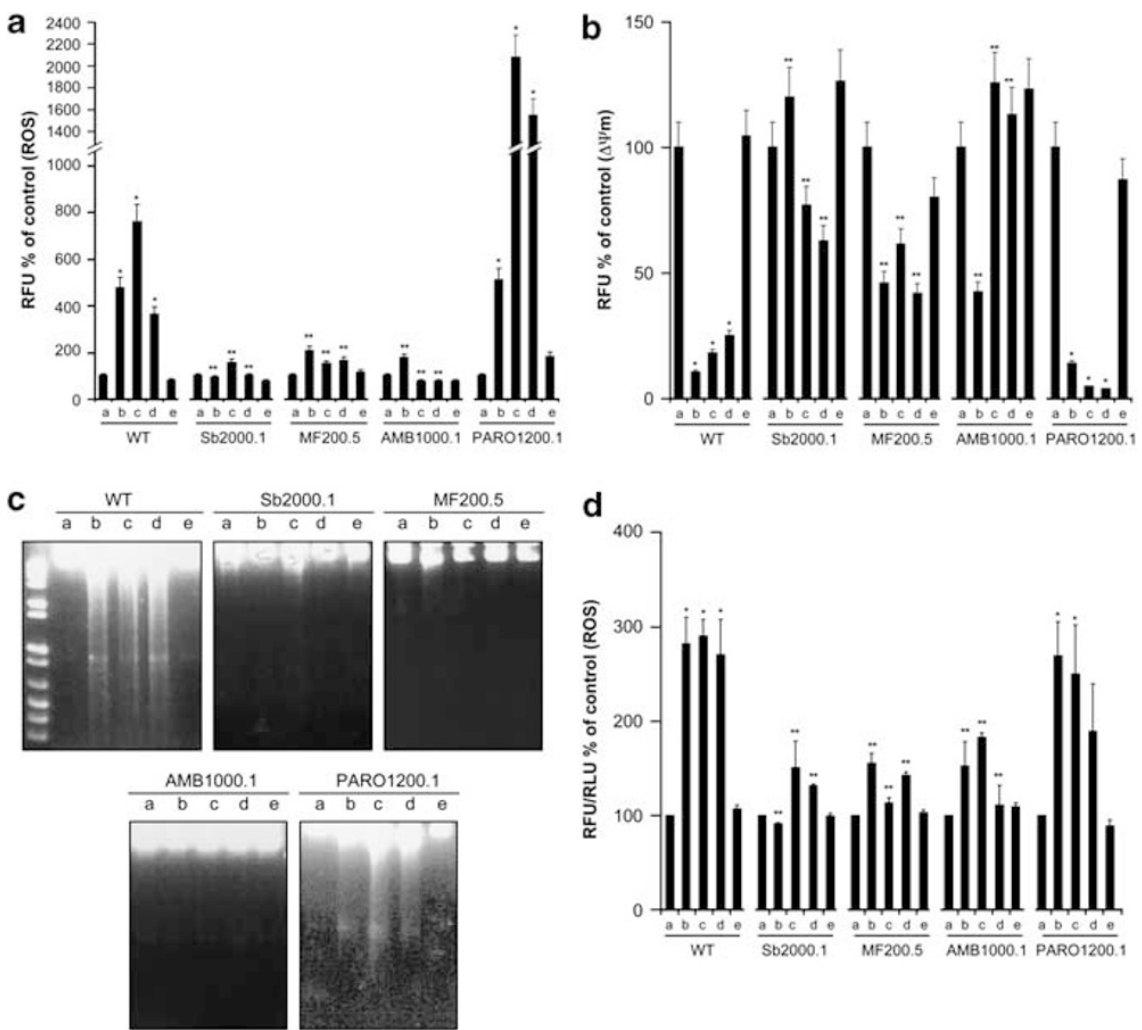

Figure 4 Cross-tolerance to drug-induced apoptosis in Leishmania drug-resistant mutants. The accumulation of ROS (a and d), loss of $\Delta \psi \mathrm{m}$ (c) and gDNA degradation (b) were measured in Leishmania WT and resistant mutants (Sb2000.1, MF200.5, AMB1000.1 and PAR01200.1). Promastigotes log-phase cultures (a, b and $\mathbf{c}$ ) were left untreated (controls, bars a) or exposed to $400 \mu \mathrm{M}$ Sblll (bars b), $25 \mu \mathrm{M} \mathrm{MF}$ (bars c), $0.2 \mu \mathrm{M}$ AMB (bars d) or $280 \mu \mathrm{M}$ PARO (bars e) for $48 \mathrm{~h}$. Aliquots were washed and incubated in the presence of (a) DCFDA (ROS) or (b) TMRE $(\Delta \psi \mathrm{m})$ for $30 \mathrm{~min}$. Fluorescence was measured at $530 \mathrm{~nm}$ (DCFDA) or $545 \mathrm{~nm}$ (TMRE) and normalized to protein concentrations. (c) Genomic DNA was analyzed on 1.3\% agarose gels MW: $1 \mathrm{~kb}$ Plus Ladder (Invitrogen). (d) Accumulation of ROS were measured in intracellular Leishmania wild-type cells and drug-resistant mutants. Purified metacyclics were preloaded with DCFDA and used to infect THP-1 macrophages. Infected cells were then left untreated (controls, bars a) or treated with $150 \mu \mathrm{M}$ of Pentostam (SbV) (bars b), $1 \mu \mathrm{M}$ of MF (bars c), $0.05 \mu \mathrm{M}$ of AMB (bars d) or $30 \mu \mathrm{M}$ of PARO (bars e). Results are the average of three independent experiments and are expressed as percentage of controls $(\mathbf{a}, \mathbf{b}$ and $\mathbf{d}) .{ }^{*} P<0.01$; ${ }^{*}$ statistically different from ${ }^{*}$ with $P<0.01$

mutational events occurred specifically in the Sb2000.1 mutant and led to increased PARO resistance (Figure 5a). This would be reminiscent of bactericidal antibiotic-induced mutagenesis in bacteria. 40

The present results suggest that Leishmania parasites resistant to SbIII, MF or AMB in the field could be more prone at acquiring multidrug resistance to these antileishmanials, while retaining sensitivity to PARO. These conclusions are supported by the observation that field isolates resistant to Sblll were less prone to undergo apoptosis following exposure to either SbIII, MF or AMB. ${ }^{7}$ A recent study also suggested the possible cross-resistance of $L$. donovani field isolates to SbIII, MF and AMB in areas of endemicity. ${ }^{41}$ Drug combination is now an important strategy in our fight against infectious diseases and this has now recently been shown to be effective in Leishmania. ${ }^{42}$ Our data provide a rationale for combining drugs with different modes of killing.

In summary, there are at least two effector mechanisms for anti-Leishmania drugs. Resistance is accompanied by tolerance to drug-induced apoptosis not only against the selective drug but also against drugs sharing a similar mode of killing. This cross-tolerance leads to a facilitated emergence of cross-resistance against other drugs that have different cellular targets but similar mode of killing.

\section{Materials and Methods}

Leishmania strains and culture conditions. L. infantum (MHOM/MA/67/ ITMAP-263) and the drug-resistant mutants $L$. infantum Sb2000.1, $L$. infantum MF200.5, L. infantum AMB1000.1 and $L$. infantum PAR01200.1 (resistant to $2000 \mu \mathrm{M}$ Sblll, $200 \mu \mathrm{M}$ MF, $1 \mathrm{mM} \mathrm{AMB}$ and $1680 \mu \mathrm{M}$ PARO, respectively) were grown in SDM-79 medium supplemented with $10 \%$ fetal bovine serum at $25^{\circ} \mathrm{C}$ Drug susceptibilities were obtained as described. Log-phase parasites were preincubated with deferoxamine $(500 \mu \mathrm{M})$ or GSH $(2 \mathrm{mM})$ for $24 \mathrm{~h}$ before being washed and subsequently exposed to drugs for $48 \mathrm{~h}$. To determine apoptotic features (ROS, $\Delta \psi \mathrm{m}$ and gDNA laddering), log-phase cultures were exposed to toxic drug concentrations for $48 \mathrm{~h}$ in order to trigger apoptosis.

Measurement of ROS accumulation. Intracellular ROS accumulation was measured using the DCFDA dye (Invitrogen, Burlington, Ontario, Canada) as described. ${ }^{28}$ Briefly, DCFDA is cell permeant and non-fluorescent. Upon its entry into live cells, the diacetate groups are cleaved by cellular esterases. Oxidation of the reduced dye occurs in the presence of ROls, causing fluorescence. After drug exposure (48 h), parasites were centrifuged at 3000 r.p.m., washed, resuspended in $500 \mu \mathrm{l}$ of Hepes- $\mathrm{NaCl}(21 \mathrm{mM}$ Hepes, $137 \mathrm{mM} \mathrm{NaCl}, 5 \mathrm{mM} \mathrm{KCl}, 0.7 \mathrm{mM}$ $\mathrm{Na}_{2} \mathrm{HPO}_{4} .7 \mathrm{H} 2 \mathrm{O}, 6 \mathrm{mM}$ glucose, $\mathrm{pH} 7.4$ ) and incubated for $30 \mathrm{~min}$ in presence of DCFDA ( $40 \mathrm{nM}$ ). After washing, $200 \mu \mathrm{l}$ of the promastigote resuspension was analyzed with a Victor fluorometer (Perkin-Elmer, Turku, Finland) at $485 \mathrm{~nm}$ excitation and $535 \mathrm{~nm}$ emission wavelengths. Fluorescence was normalized with proteins concentration measured using the 2D QuantKit (GE Healthcare, Quebec, Canada). A similar procedure was used with the MitoSOX dye (Invitrogen, Ontario, Canada) to determine the ROS accumulation occurring within the mitochondrion. MitoSOX is a live-cell permeant, and rapidly and selectively targets the 

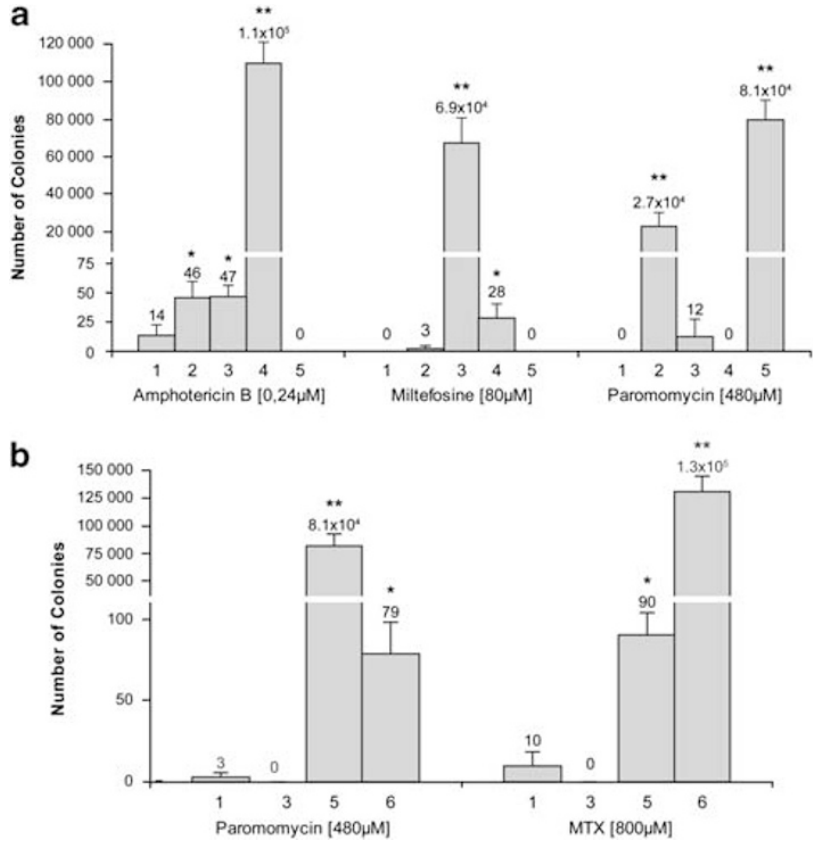

Figure 5 Accelerated selection of multidrug-resistant Leishmania cells. Wildtype parasites or drug-resistant mutants $\left(5 \times 10^{6}\right.$ cells) were plated on drugcontaining SDM-agar plates and colonies were counted after 14 days. (a) Wild-type (WT) cells and mutant cell lines Sb2000.1, MF200.5, AMB1000.1 and PAR01200.1 were plated on AMB-, MF- and PARO-containing plates. (b) Wild-type cells and mutant cell lines MF200.5, PAR01200.1 and MTX1000.3 were plated on PAROand MTX-containing plates. Results are the average of three independents experiments. 1, WT; 2, Sb2000.1; 3, MF200.5; 4, AMB1000.1; 5, PAR01200.1; 6, MTX1000.1. ${ }^{*} P<0.05 ;{ }^{* *} P<0.01$

mitochondria. Once in the mitochondria, the MitoSOX probe is oxidized by superoxide, hence, exhibiting a red fluorescence $(580 \mathrm{~nm})$.

Measurement of ROS accumulation in intracellular amastigotes. Luciferase-containing promastigotes were harvested in stationary growth phase (6 days), and metacyclics were purified using the peanut agglutinin assay. ${ }^{43}$ Briefly, $10 \mathrm{ml}$ of stationary-phase cultures were centrifuged at 3000 r.p.m. washed and resuspended in $10 \mathrm{ml}$ Hepes- $\mathrm{NaCl}$ containing $100 \mu \mathrm{g} / \mathrm{ml}$ of the lectin peanut agglutinin (Sigma-Aldrich, Ontario, Canada), and incubated with agitation for $30 \mathrm{~min}$ at room temperature. The mixture was then centrifuged at 900 r.p.m.the metacyclic-containing supernatants were collected and centrifuged at 3000 r.p.m., and the pellet was washed and resuspended in appropriate Hepes- $\mathrm{NaCl}$ volume. Purified metacyclics were preincubated with $40 \mathrm{nM}$ of DCFDA for $20 \mathrm{~min}$, washed and used for subsequent macrophages infection as described. ${ }^{44} \mathrm{At} 3 \mathrm{~h}$ after infection, macrophages were washed to remove non-phagocytized parasites. Drugs at $\mathrm{EC}_{50}$ levels: Pentostam $(\mathrm{SbV}, 150 \mu \mathrm{M}), \mathrm{MF}(1 \mu \mathrm{M})$, AMB $(0.05 \mu \mathrm{M})$ or PARO $(30 \mu \mathrm{M})$ were added. After 2 days, DCFDA fluorescence was taken to measure oxidants accumulation within the amastigotes parasites. Macrophages were then lysed and processed in order to measure luminescence from luciferase-expressing amastigotes as a measure of survival to normalize oxidants-induced fluorescence.

Measurement of $\Delta \psi \mathrm{m}$ and gDNA laddering. $\Delta \psi \mathrm{m}$ was measured using the cationic lipophilic fluorescent TMRE dye as described. ${ }^{7}$ Fluorescence was normalized with proteins concentrations as described above. gDNA was harvested from untreated and treated parasites using a method previously described. ${ }^{7}$ In all, $10 \mu \mathrm{g}$ of gDNA was loaded on a $1.3 \%$ agarose gel and was allowed to migrate at $90 \mathrm{~V}$ for $1 \mathrm{~h}$

Mitochondrial localization of drug-induced ROS using fluorescence microscopy. After drug exposure $(48 \mathrm{~h})$, parasites were centrifuged at 3000 r.p.m., washed and resuspended in $500 \mu \mathrm{l}$ of Hepes- $\mathrm{NaCl}$, and were coincubated for $30 \mathrm{~min}$ in presence of both DCFDA ( $40 \mathrm{nM}$ ) and Mitotracker
$(40 \mathrm{nM})$. Parasite were subsequently centrifuged at 3000 r.p.m., washed and resuspended in $500 \mu \mathrm{l}$ of Hepes- $\mathrm{NaCl}$, and $20 \mu \mathrm{l}$ of parasite suspension was layered on poly-L-lysine-coated microscope slides under a cover slips. Slides were analyzed using a fluorescence microscope (Nikon eclipse TE300 fluorescence microscope; Nikon, Ontario, Canada) using typical green and red filters. Images were captured using Image Pro Plus 5.0 software (Media Cybernetics, Inc., Bethesda, MD, USA). At least 20 microscopic fields were observed for each sample.

Cross-resistance selection on drug-containing SDM-agar plates. Equal number $\left(5 \times 10^{6}\right)$ of wild-type or drug-resistant parasites were centrifuged at 3500 r.p.m., washed two times in Hepes- $\mathrm{NaCl}$ and resuspended in $50 \mu \mathrm{l}$ Hepes- $\mathrm{NaCl}$, and subsequently plated on SDM-agar plates containing either $0.24 \mu \mathrm{M}$ of $\mathrm{AMB}, 80 \mu \mathrm{M}$ of MF or $480 \mu \mathrm{M}$ of PARO. These concentrations, which corresponded to six times the $\mathrm{EC}_{50}$ of the wild-type strain, were the lowest allowing minimal growth of wild-type parasites. Colonies were counted after 14 days. Plating was repeated at least three independent times.

\section{Conflict of Interest}

The authors declare no conflict of interest.

Acknowledgements. We thank Drs. Danielle Légaré and Richard Poulin for critical reading of the manuscript. This work was funded in part by a ClHR group grant and operating grants to MO. WM is a Strategic Training Fellow of the Strategic Training Program in Microbial Resistance, a partnership of the ClHR Institute of Infection and Immunity and the Fonds de la Recherche en Santé du Québec. MO holds the Canada Research Chair in Antimicrobial Resistance.

1. Kohanski MA, Dwyer DJ, Collins JJ. How antibiotics kill bacteria: from targets to networks. Nat Rev Microbiol 2010; 8: 423-435.

2. Baguley BC. Multidrug resistance in cancer. Methods Mol Biol 2010; 596: 1-14.

3. Dive C, Hickman JA. Drug-target interactions: only the first step in the commitment to a programmed cell death? Br J Cancer 1991; 64: 192-196.

4. Mehta A, Shaha $C$. Mechanism of metalloid-induced death in Leishmania spp.: role of iron, reactive oxygen species, Ca2t, and glutathione. Free Radic Biol Med 2006; 40: 1857-1868.

5. Roy A, Ganguly A, BoseDasgupta S, Das BB, Pal C, Jaisankar P et al. Mitochondriadependent reactive oxygen species-mediated programmed cell death induced by $3,3^{\prime}$ diindolylmethane through inhibition of F0F1-ATP synthase in unicellular protozoan parasite Leishmania donovani. Mol Pharmacol 2008; 74: 1292-1307.

6. Sen R, Bandyopadhyay S, Dutta A, Mandal G, Ganguly S, Saha P et al. Artemisinin triggers induction of cell-cycle arrest and apoptosis in Leishmania donovani promastigotes. $J$ Med Microbiol 2007; 56: 1213-1218.

7. Vergnes B, Gourbal B, Girard I, Sundar S, Drummelsmith J, Ouellette M. A proteomics screen implicates HSP83 and a small kinetoplastid calpain-related protein in drug resistance in Leishmania donovani clinical field isolates by modulating drug-induced programmed cell death. Mol Cell Proteomics 2007; 6: 88-101.

8. Zangger H, Mottram JC, Fasel N. Cell death in Leishmania induced by stress and differentiation: programmed cell death or necrosis? Cell Death Differ 2002; 9: 1126-1139.

9. Ouellette M, Drummelsmith J, Papadoulou B. Leishmaniasis: drugs in the clinic, resistance and new developments. Drug Resist Updat 2004; 7: 257-266.

10. Sundar S, Jha TK, Thakur CP, Sinha PK, Bhattacharya SK. Injectable paromomycin for Visceral leishmaniasis in India. N Engl J Med 2007; 356: 2571-2581.

11. Baiocco P, Colotti G, Franceschini S, Ilari A. Molecular basis of antimony treatment in leishmaniasis. J Med Chem 2009; 52: 2603-2612.

12. Berman JD, Gallalee JV, Best JM. Sodium stibogluconate (Pentostam) inhibition of glucose catabolism via the glycolytic pathway, and fatty acid beta-oxidation in Leishmania mexicana amastigotes. Biochem Pharmacol 1987; 36: 197-201.

13. Chakraborty AK, Majumder HK. Mode of action of pentavalent antimonials: specific inhibition of type I DNA topoisomerase of Leishmania donovani. Biochem Biophys Res Commun 1988; 152: 605-611.

14. Cunningham ML, Fairlamb AH. Trypanothione reductase from Leishmania donovani. Purification, characterisation and inhibition by trivalent antimonials. Eur J Biochem 1995; 230: $460-468$.

15. Lucumi A, Robledo S, Gama V, Saravia NG. Sensitivity of Leishmania viannia panamensis to pentavalent antimony is correlated with the formation of cleavable DNA-protein complexes. Antimicrob Agents Chemother 1998; 42: 1990-1995.

16. Sereno D, Holzmuller P, Mangot I, Cuny G, Ouaissi A, Lemesre JL. Antimonial-mediated DNA fragmentation in Leishmania infantum amastigotes. Antimicrob Agents Chemother 2001; 45: 2064-2069. 
17. Sudhandiran G, Shaha C. Antimonial-induced increase in intracellular Ca2+ through non-selective cation channels in the host and the parasite is responsible for apoptosis of intracellular Leishmania donovani amastigotes. J Biol Chem 2003; 278 : 25120-25132

18. Wyllie S, Cunningham ML, Fairlamb AH. Dual action of antimonial drugs on thiol redox metabolism in the human pathogen Leishmania donovani. J Biol Chem 2004; 279: 39925-39932

19. Lux H, Heise N, Klenner T, Hart D, Opperdoes FR. Ether-lipid (alkyl-phospholipid) metabolism and the mechanism of action of ether-lipid analogues in Leishmania. Mol Biochem Parasitol 2000; 111: 1-14.

20. Rakotomanga M, Blanc S, Gaudin K, Chaminade P, Loiseau PM. Miltefosine affects lipid metabolism in Leishmania donovani promastigotes. Antimicrob Agents Chemother 2007; 51: $1425-1430$.

21. Ramos H, Valdivieso E, Gamargo M, Dagger F, Cohen BE. Amphotericin B kills unicellular leishmanias by forming aqueous pores permeable to small cations and anions. $J$ Membr Biol 1996; 152: 65-75.

22. Goad LJ, Holz Jr GG, Beach DH. Sterols of Leishmania species. Implications for biosynthesis. Mol Biochem Parasitol 1984; 10: 161-170.

23. Fernandez MM, Malchiodi EL, Algranati ID. Differential effects of paromomycin on ribosomes of Leishmania mexicana and mammalian cells. Antimicrob Agents Chemother 2011; 55: 86-93

24. Maarouf M, de Kouchkovsky $Y$, Brown S, Petit PX, Robert-Gero M. In vivo interference of paromomycin with mitochondrial activity of Leishmania. Exp Cell Res 1997; 232 339-348.

25. Maarouf M, Lawrence F, Croft SL, Robert-Gero M. Ribosomes of Leishmania are a target for the aminoglycosides. Parasitol Res 1995; 81: 421-425.

26. Paris C, Loiseau PM, Bories C, Breard J. Miltefosine induces apoptosis-like death in Leishmania donovani promastigotes. Antimicrob Agents Chemother 2004; 48 852-859.

27. Verma NK, Dey CS. Possible mechanism of miltefosine-mediated death of Leishmania donovani. Antimicrob Agents Chemother 2004; 48: 3010-3015.

28. Moreira W, Leblanc $E$, Ouellette $M$. The role of reduced pterins in resistance to reactive oxygen and nitrogen intermediates in the protozoan parasite Leishmania. Free Radic Biol Med 2009; 46: 367-375.

29. Arnoult D, Akarid K, Grodet A, Petit PX, Estaquier J, Ameisen JC. On the evolution of programmed cell death: apoptosis of the unicellular eukaryote Leishmania major involves cysteine proteinase activation and mitochondrion permeabilization. Cell Death Differ 2002; 9: $65-81$.

30. Nare B, Luba J, Hardy LW, Beverley S. New approaches to Leishmania chemotherapy: pteridine reductase 1 (PTR1) as a target and modulator of antifolate sensitivity. Parasitology 1997; 114(Suppl): S101-S110.

31. Ouellette M, Drummelsmith J, El-Fadili A, Kundig C, Richard D, Roy G. Pterin transport and metabolism in Leishmania and related trypanosomatid parasites. Int J Parasitol 2002; 32: 385-398.
32. Mukhopadhyay P, Rajesh M, Hasko G, Hawkins BJ, Madesh M, Pacher P. Simultaneous detection of apoptosis and mitochondrial superoxide production in live cells by flow cytometry and confocal microscopy. Nat Protoc 2007; 2: 2295-2301.

33. Kohanski MA, Dwyer DJ, Hayete B, Lawrence CA, Collins JJ. A common mechanism of cellular death induced by bactericidal antibiotics. Cell 2007; 130: 797-810.

34. Kroemer G, Galluzzi L, Vandenabeele P, Abrams J, Alnemri ES, Baehrecke EH et a. Classification of cell death: recommendations of the Nomenclature Committee on Cell Death 2009. Cell Death Differ 2009; 16: 3-11.

35. Smirlis D, Duszenko M, Ruiz AJ, Scoulica E, Bastien P, Fasel N et al. Targeting essential pathways in trypanosomatids gives insights into protozoan mechanisms of cell death. Parasit Vectors 2010; 3: 107.

36. Croft SL, Sundar S, Fairlamb AH. Drug Resistance in Leishmaniasis. Clin Microb Rev 2006; 19: 111-126.

37. Ouellette M, Drummelsmith J, Papadopoulou B. Leishmaniasis: drugs in the clinic resistance and new developments. Drug Resist Updat 2004; 7: 257-266.

38. Mukhopadhyay R, Dey S, Xu N, Gage D, Lightbody J, Ouellette M et al. Trypanothione overproduction and resistance to antimonials and arsenicals in Leishmania. Proc Natl Acad Sci USA 1996; 93: 10383-10387.

39. Wyllie S, Mandal G, Singh N, Sundar S, Fairlamb AH, Chatterjee M. Elevated levels of tryparedoxin peroxidase in antimony unresponsive Leishmania donovani field isolates. Mol Biochem Parasitol 2010; 173: 162-164.

40. Kohanski MA, DePristo MA, Collins JJ. Sublethal antibiotic treatment leads to multidrug resistance via radical-induced mutagenesis. Mol Cell 2010; 37: 311-320.

41. Kumar D, Kulshrestha A, Singh R, Salotra P. In vitro susceptibility of field isolates of Leishmania donovani to Miltefosine and Amphotericin B: correlation with sodium antimony gluconate susceptibility and implications for treatment in areas of endemicity. Antimicrob Agents Chemother 2009; 53: 835-838.

42. Chappuis F, Sundar S, Hailu A, Ghalib H, Rijal S, Peeling RW et al. Visceral leishmaniasis: What are the needs for diagnosis, treatment and control? Nat Rev Microbiol 2007; 5 873-882.

43. Sacks DL, Perkins PV. Development of infective stage Leishmania promastigotes within phlebotomine sand flies. Am J Trop Med Hyg 1985; 34: 456-459.

44. Mukherjee A, Roy G, Guimond C, Ouellette M. The gamma-glutamylcysteine synthetase gene of Leishmania is essential and involved in response to oxidants. Mol Microbiol 2009; 74: $914-927$

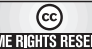

Cell Death and Disease is an open-access journal published by Nature Publishing Group. This work is licensed under the Creative Commons Attribution-Noncommercial-No Derivative Works 3.0 Unported License. To view a copy of this license, visit http://creativecommons.org/licenses/by-nc-nd/3.0/

\section{Supplementary Information accompanies the paper on Cell Death and Disease website (http://www.nature.com/cddis)}

